\title{
Magnetic order and instability in newly synthesized CoSeAs marcasite
}

\author{
Yiyao Chen, ${ }^{1}$ G. Yumnam $\odot,{ }^{1}$ A. Dahal, ${ }^{1}$ J. A. Rodriguez-Rivera $\odot,{ }^{2,3}$ Guangyong Xu, ${ }^{2}$ T. W. Heitmann, ${ }^{4}$ and D. K. Singh ${ }^{1, *}$ \\ ${ }^{1}$ Department of Physics and Astronomy, University of Missouri, Columbia, Missouri 65211, USA \\ ${ }^{2}$ National Institute of Standards and Technology, Gaithersburg, Maryland 20899, USA \\ ${ }^{3}$ Department of Materials Sciences and Engineering, University of Maryland, College Park, Maryland 20742, USA \\ ${ }^{4}$ University of Missouri Research Reactor, Columbia, Missouri 65211, USA
}

(Received 26 December 2019; revised manuscript received 3 March 2020; accepted 8 April 2020; published 13 May 2020)

\begin{abstract}
Marcasite class of compounds provide a facile platform to explore novel phenomena of fundamental and technological importance, such as unconventional superconductivity or high-performance electrocatalyst. We report the synthesis and experimental investigation of a marcasite CoSeAs in this paper. Experimental investigation of this material using neutron scattering measurements reveals weak magnetic correlation of cobalt ions below $T=36.2 \mathrm{~K}$. The modest isotropic exchange interaction between cobalt moments, inferred from random phase approximation analysis, hints of a magnetically unstable environment. It is a desirable characteristic to induce unconventional superconductivity via chemical pressure application.
\end{abstract}

DOI: 10.1103/PhysRevResearch.2.023168

\section{INTRODUCTION}

Transition metals are at the core of many interesting phenomena in magnetism [1,2]. Among the many chemical groups of transition metal compounds, marcasite phase is one of the most intriguing lattice groups with a $\mathrm{FeS}_{2}$-type crystal structure $[3,4]$. These materials are of strong technological importance, especially for application in photovoltaics and in the design of efficient electrocatalysts. Some notable examples include the demonstration of high-performance electrocatalyst in $\mathrm{FeX}_{2}(X=\mathrm{S}, \mathrm{Se})$ and high absorption coefficient photovoltaic property in $\mathrm{CoSe}_{2}$ [5-7]. Marcasites are also known to manifest superconducting phenomena of both conventional and unconventional origins [8,9]. The proposed observation of unconventional superconductivity in marcasite phase $\mathrm{FeBi}_{2}$ is attributed to the competing instability of underlying magnetism in the system [9]. The chemical structure of a marcasite is described by the distorted octahedrons of six anions (ligands) enveloping the cation of $3 \mathrm{~d}$ or $4 \mathrm{~d}$ orbital transition metal ion [10]. Despite the presence of the transition metal as the key constituting element in the stoichiometric composition, most of them are either diamagnetic or paramagnetic with semiconducting electrical characteristics [11]. The Jahn-Teller distortion in the transition metal ion coordination octahedron is arguably responsible for the nonmagnetic ground state in a majority of the transition metal marcasites [8]. We have synthesized another compound, CoSeAs, in this series. Detailed experimental investigations of CoSeAs using

\footnotetext{
*singhdk@missouri.edu

Published by the American Physical Society under the terms of the Creative Commons Attribution 4.0 International license. Further distribution of this work must maintain attribution to the author(s) and the published article's title, journal citation, and DOI.
}

elastic and inelastic neutron scattering measurements suggest the development of long-range magnetic order below $T_{c}=$ $36.2 \mathrm{~K}$. It sets a precedent in 1:1:1 stoichiometric composition of the corresponding lattice group. Furthermore, we find that $\mathrm{Co}$ ions are correlated with the weak nearest neighbor exchange interaction, $J=0.25$ (4) $\mathrm{meV}$, which makes the system susceptible to a transition to nonmagnetic or different phase of matter, such as superconductivity, under modest external effect.

CoSeAs stands at the crossroads of CoSe and CoAs compounds that crystallize in a MnP-type tetragonal structure. While $\mathrm{CoSe}$ is argued to manifest a combination of ferromagnetic and spin glass properties [12,13], CoAs is considered nonmagnetic [14]. However, both materials exhibit the metallic characteristic. Sharing magnetic traits of both compounds, CoSeAs is on the verge of magnetic instability. CoSeAs crystallizes in the $\mathrm{FeS}_{2}$-type marcasite structure with weak metallic characteristic, bordering to the semiconducting phase at low temperature. We have synthesized the polycrystalline samples of CoSeAs using repetitive solid-state reaction method in evacuated quartz tubes. The starting materials were $99.998 \%$ Co, $99.999 \%$ Se (Alfa Aesar), and $99.997 \%$ As (Sigma-Aldrich) [15]. CoSe was synthesized from the stoichiometric composition of Co and Se. The mixture was grinded, pelletized, and loaded into a quartz tube, subsequently evacuated and sealed, then annealed at $900^{\circ} \mathrm{C}$ for two days. After confirming the pure structure of CoSe (with no oxidation) using x-ray diffraction (XRD) measurement, stoichiometric amount of As was added to CoSe powder. The mixture was ground, pelletized, and sintered in evacuated quartz tube at $900^{\circ} \mathrm{C}$ for another two days. As shown in Fig. 1, the XRD pattern clearly manifests the high quality of the synthesized polycrystalline CoSeAs. X-ray peaks are well indexed by the Pnnm space group body centered orthorhombic $\mathrm{FeS}_{2}$-type marcasite structure, as shown in the inset in Fig. 1, with lattice parameters of $a=4.756 \AA, b=5.756 \AA$, and $c=3.570 \AA$. 


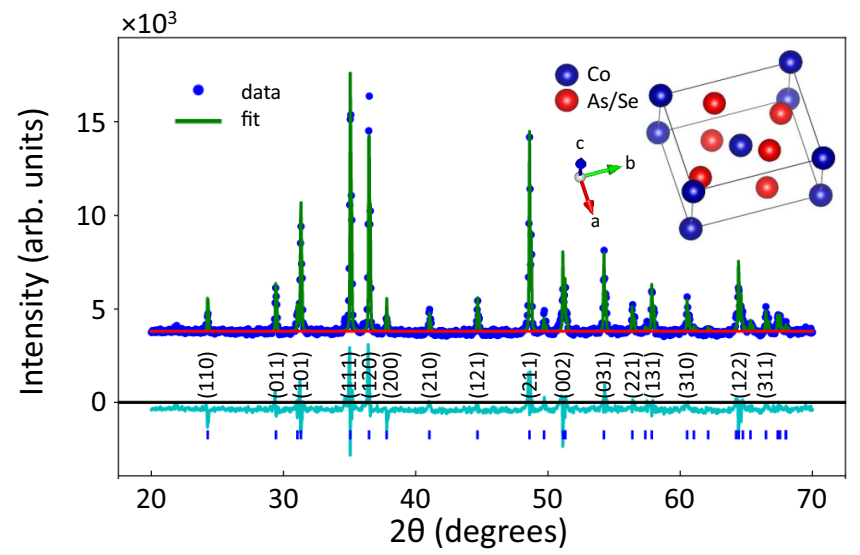

FIG. 1. Crystal structure of CoSeAs marcasite and X-ray diffraction data. X-ray diffraction pattern of as synthesized CoSeAs. XRD peaks are indexed with the $\mathrm{FeS}_{2}$-type marcasite structure. Inset shows the crystal structure of CoSeAs compound.

\section{RESULTS AND DISCUSSION}

There is not much known about CoSeAs. The knowledge of electrical conducting properties and a theoretical understanding of the density of states at the Fermi surface are necessary to characterize this material. We have performed first principles electronic structure calculations for CoSeAs based on the density functional theory by employing the plane-wave basis set, as implemented in QUANTUM-ESPRESSO [16]. The projector augmented wave method was used with Troullier-Martins norm-conserving pseudopotential with nonlinear core correction. The calculations were performed with (without) spin-orbit interaction by using fully (scalar) relativistic pseudopotentials. The exchange correlation functional was treated within the generalized gradient approximation of Perdew-Burke-Ernzerhof (PBE-GGA) [17]. The correlation effects of Co $3 d$ electrons were included via GGA + $\mathrm{U}$ method within the simplified rotational invariant scheme of Cococcioni et al. [18]. The value of on-site Coulombic interaction term (U) was set to a well-tested value of $U=$ $4.5 \mathrm{eV}$. A well-converged kinetic-energy cutoff of $80 \mathrm{Ry}$ was used with a Monkhorst-Pack sampling of $16 \times 16 \times 16$. The experimental lattice parameter obtained from $x$-ray diffraction measurements were used as the initial configuration of the atoms for the DFT calculations. The magnetic configuration of the Co atoms was initialized to be ferromagnetic. Note that the final magnetic configuration of Co atoms is independent of the initial magnetization direction. A strict self-consistent energy convergence criterion of $10^{-8}$ Ry was imposed. As shown in Fig. 2(c), the calculated spin-resolved density of states (DOS) show that the characteristic Co- $d$ states are embedded well below the Fermi energy for both majority [Fig. 2(a)] and minority [Fig. 2(b)] spin carriers, indicating weak-metallic or semiconducting characteristic due to the embedded Co $d$ orbitals. We note that the minority-spin carriers exhibit a much larger DOS at the Fermi level than the majority-spin carriers, which is a typical characteristic of ferromagnetic material. This is further substantiated by the spin-resolved hole Fermi surface, as shown in Fig. 2(b), where the minority-carrier hole Fermi surface is more populated (a)

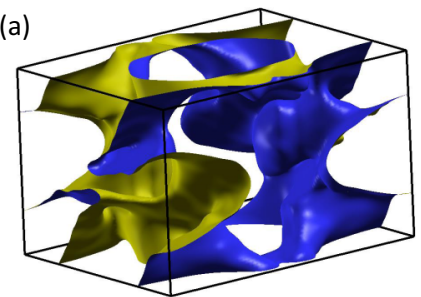

(c)
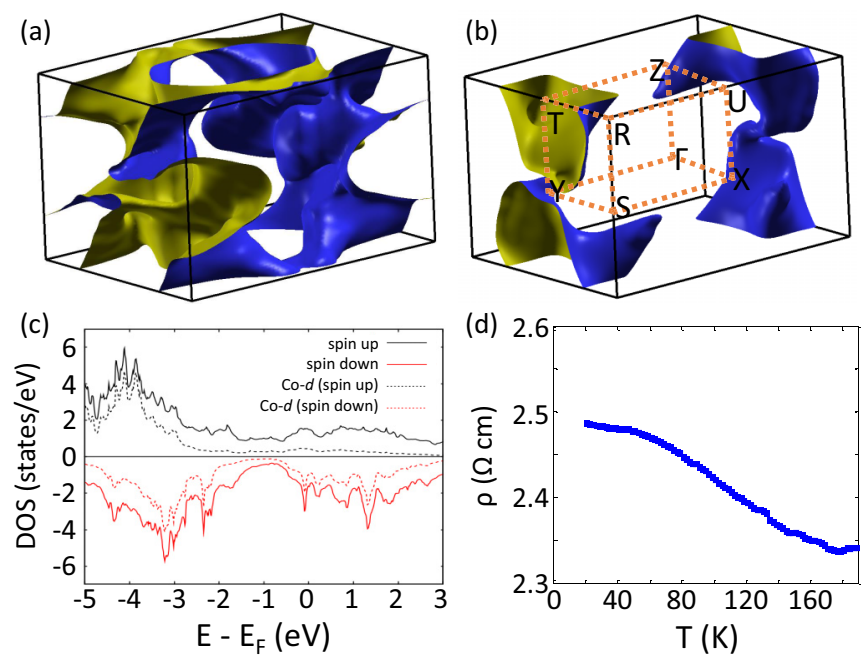

(d) 2.6

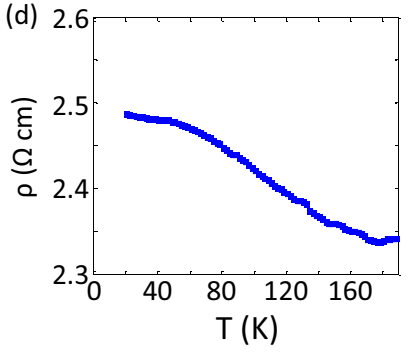

FIG. 2. Fermi surface and electrical characteristic of CoSeAs. [(a), (b)] Spin resolved Fermi surfaces of CoSeAs: spin up (a) and spin down (b). (c) Density of states calculated using density functional theory (see text) elucidates the semiconducting character of the material. (d) Electrical measurement shows very weak metallic conductivity at high temperature. The system manifests semiconducting behavior at lower temperature, which is in agreement with electrical properties of marcasite phase compounds.

even though it has a much smaller volume compared to that of the majority carriers [Fig. 2(a)]. It gives rise to nondegenerate energies of electrons with opposite spins.

The high-quality polycrystalline sample of CoSeAs was electrically characterized using a closed-cycle refrigeratorbased cryostat with a base temperature of $T \simeq 5 \mathrm{~K}$. As shown in Fig. 2(d), the system exhibits very weak metallicto-semiconducting behavior as temperature is reduced, also consistent with the DFT calculations. Information about the underlying magnetic properties is obtained from detailed elastic and inelastic neutron scattering measurements. Neutron measurements were performed on a 4.4-g polycrystalline sample of CoSeAs at the multiaxis crystal spectrometer (MACS) with fixed final neutron energy of $E_{f}=3.7 \mathrm{meV}$ at the NIST Center for Neutron Research. Additional measurements were performed on the spin-polarized triple-axis spectrometer (SPINS) at NCNR and a position-sensitive detector (PSD) powder diffractometer at the University of Missouri Research Reactor with fixed final neutron energy of $37 \mathrm{meV}$ using graphite monochromator. Elastic measurements at SPINS employed a flat pyrolytic graphite (PG) analyzer with cold $\mathrm{BeO}$ filter in front, while the measurements on the powder diffractometer was performed using the tighter collimations before the monochromator and a PG filter. Inelastic measurements on MACS were performed in the focused analyzer configuration with fixed $E_{F}=3.7 \mathrm{meV}$ and the energy resolution of $\simeq 0.25 \mathrm{meV}$. The sample was loaded in liquid ${ }^{4} \mathrm{He}$ cooled cryostat with the lowest accessible temperature of $T=1.7 \mathrm{~K}$.

Elastic scattering measurements on CoSeAs powder are used to infer the underlying static magnetic correlation between Co ions. We show the representative scans at two temperatures in Fig. 3(a). Additional Bragg peaks arise as the sample is cooled to low temperature, indicating the 

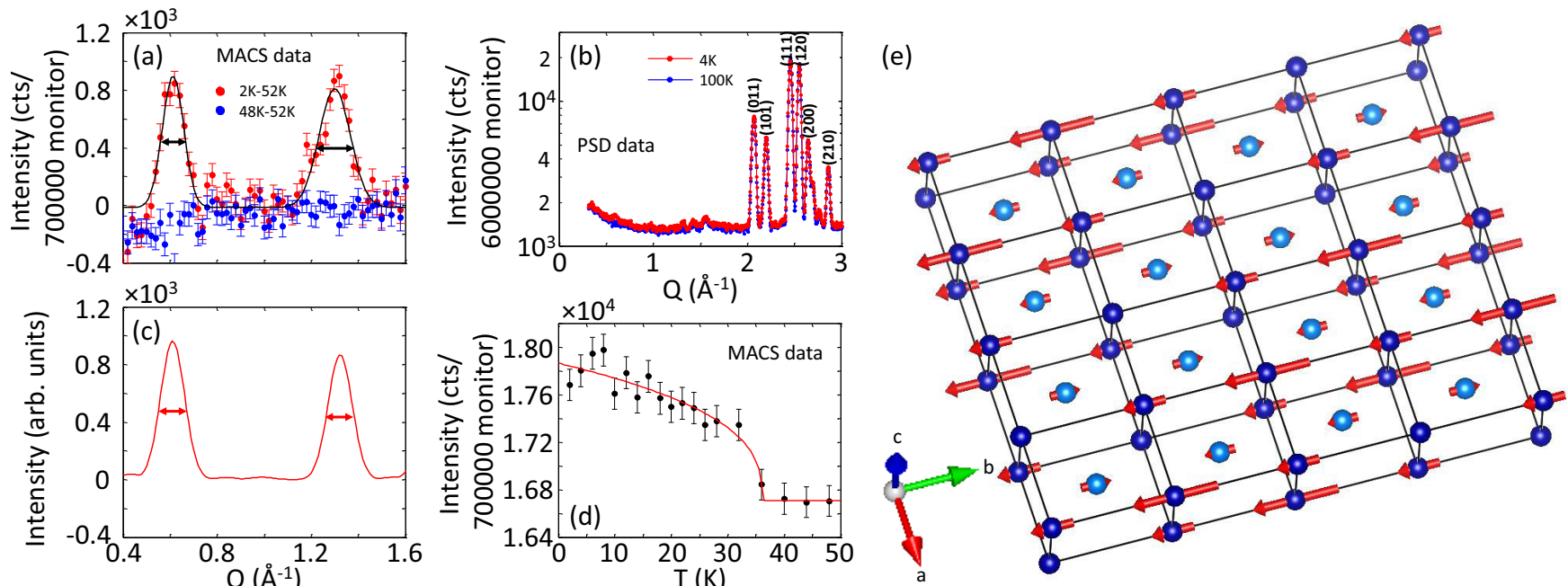

FIG. 3. Elastic neutron scattering measurements of CoSeAs and order parameter as a function of temperature. (a) $2 \theta$ scans (converted into absolute wave vector $q$ ) at different temperatures, obtained on MACS spectrometer. Experimental results were also reproduced on SPINS spectrometer. Long monitor count was implemented to resolve the weak underlying magnetism in the system. Magnetic Bragg peaks, identified as $(1 / 41 / 41 / 4)$ (equivalent to $q=0.6 \AA^{-1}$ ) and (100) (equivalent to $q=1.32 \AA^{-1}$ ) in the figure, emerge at low $q$ as temperature reduces. Experimental data are well described by resolution-limited Gaussian curve. (b) $2 \theta$ scans spanning a broader $q$ range, performed at PSD instrument at MURR. Magnetic peaks are about ten times weaker than the weakest nuclear peak. (c) Numerically simulated peak intensity for the spin structure, shown in panel (e), is used to construct the Gaussian profile by utilizing the instrument's $q$ resolution. Calculated value is in good agreement with experimental results. (d) Intensity vs temperature at magnetic $q=0.595 \AA^{-1}$. Magnetic order transition temperature of $T=36.2 \mathrm{~K}$ is deduced by fitting the order parameter plot using power law. (e) Spin configuration of Co ions in CoSeAs, figure produced in VESTA software [19]. In all plots, error bars represent one standard deviation.

development of magnetic order in the system. Compared to the nuclear peak intensities, shown in Fig. 3(b), magnetic peaks are significantly weaker. Furthermore, elastic measurements required long counting time to obtain the statistically significant magnetic peak intensities. Together, they hint of small ordered moment of Co ions in the system [20]. Experimental data are well described by the resolution-limited Gaussian line shape. The estimated full width half maximum (FWHM) of magnetic peak is comparable to the instrument resolution of the MACS spectrometer, suggesting the existence of long-range magnetic order in CoSeAs. The magnetic order is found to persist to reasonably high temperature. In Fig. 3(d), we show the plot of order parameter as a function of temperature at the magnetic wave vector $q=0.595 \AA^{-1}$. Fitting of experimental data using a power law, given by $I \propto\left(1-T / T_{c}\right)^{-\beta}$ [21], yields a transition temperature of $T_{c}=36.2 \mathrm{~K}$ to magnetic ordered state. The estimated value of power law exponent is $\beta=0.357(4)$.

The magnetic wave vectors are identified to be both integer and rational fractions of reciprocal lattice units, e.g., (100) and $(1 / 41 / 41 / 4)$. The nature of magnetic correlation is deduced by performing detailed numerical modeling of experimental data. The experimentally observed structure factor, estimated from the Gaussian fit of the elastic data, are compared with the numerically calculated structure factor for model spin configurations. Structure factor is calculated using, $F_{M}=$ $\sum_{j} S_{\perp j} p_{j} e^{i Q r_{j}} e^{-W_{j}}$ [22], where $S_{\perp}=\hat{Q} \times(S \times \hat{Q})$ is the spin component perpendicular to the $Q, p=\left(\frac{\gamma r_{0}}{2}\right) g f(Q),\left(\frac{\gamma r_{0}}{2}\right)=$ $0.2695 \times 10^{-12} \mathrm{~cm}, g$ is the Lande splitting factor and was taken to be $g=2, f(Q)$ is the magnetic form factor, and $e^{-W_{j}}$ is the Debye-Waller factor and was taken to be $1[22,23]$.
Simulated intensities are powder averaged by multiplying with an appropriate factor of $[1 / \sin (\theta) \cos (2 \theta)]$ [22]. Best fit to experimental data is obtained for magnetic moment arrangements composed of two magnetic sublattices: (a) Co ions occupying the vertices of the orthorhombic lattice are ferromagnetically aligned along the $b$ axis and arranged in a density wave configuration with quadrupled magnetic unit cell, and (b) Co moments at the body-centered position are arranged in a density wave configuration with quadrupled magnetic unit cell (see details in the Supplemental Material [24]). As shown in Fig. 3(c), the numerically simulated powder profile for the aforementioned spin structure, shown in Fig. 3(e), well describes the experimentally observed diffraction pattern. The proposed spin configuration also gives rise to peak intensity at (001) rlu, which was not resolved in the experimental data. The estimated ordered moment is $0.26(5) \mu_{B}$. Such a small value of the ordered moment reflects the nearly compensated spin polarities in individual anion octahedron. This is consistent with the general observation in marcasite phase magnetic material of weak or no magnetic order due to the strong screening of magnetic moment by conduction electrons. Experimental findings hint of the weakly correlated Co ions in CoSeAs.

To gain insight about the strength of exchange interaction between $\mathrm{Co}$ ions in CoSeAs, we have performed detailed inelastic measurements. In Fig. 4(a), we show the color map of inelastic spectrum, obtained on a MACS spectrometer, at $T=1.7 \mathrm{~K}$. A $q$-independent band of inelastic excitation tends to develop below $E \simeq 3 \mathrm{meV}$ at low temperature. The excitation at higher $q$ follows the Co form factor, thus gradually weakens. Inelastic data are background subtracted and 

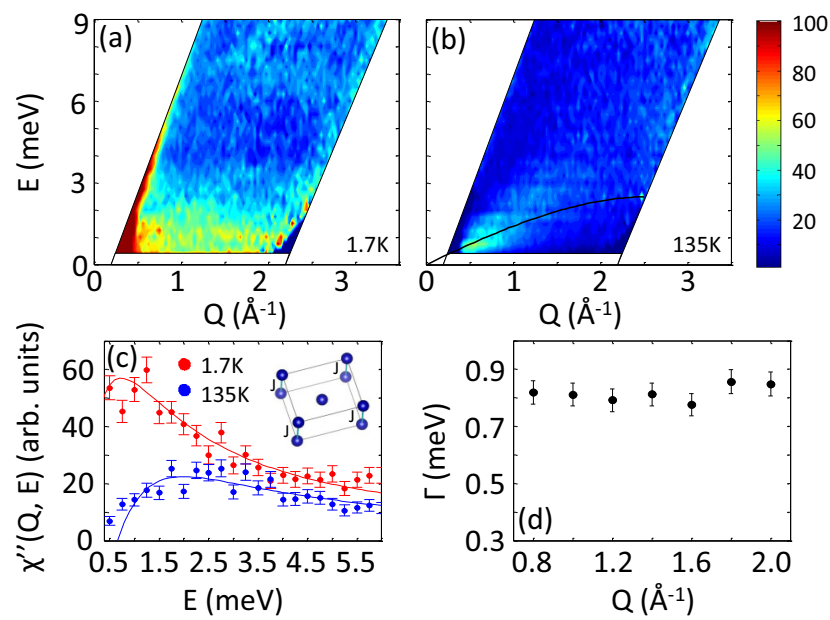

FIG. 4. Inelastic measurements of CoSeAs. [(a), (b)] 2D map of energy momentum, obtained on MACS spectrometer, at $T=1.7$ and $135 \mathrm{~K}$, respectively. Experimental data are background subtracted (by measuring $\mathrm{Al}$ in identical experimental conditions) and thermally balanced. Broad $q$-independent excitation is detected at $E \leqslant 3 \mathrm{meV}$ at low temperature. At a much higher temperature above magnetic ordering transition, the broad excitation disappears. Rather, a phonon dispersion tends to emerge (a curve is drawn for guide to the eye). (c) Plot of dynamic susceptibility vs energy at fixed $q=1.4 \AA^{-1}$ at low and high temperatures. Fitting using RPA model reveals clear peak-type structure at $E \simeq 0.75 \mathrm{meV}$ at $T=1.7 \mathrm{~K}$ (see text for detail). (d) Estimated $\Gamma$ vs q at $T=1.7 \mathrm{~K}$, manifesting $q$-independent characteristic of the dynamic behavior.

thermally balanced by multiplying the intensity by a factor of $\pi\left[1-\exp \left(-E / k_{B} T\right)\right]$. The absence of any dispersion in experimental data suggests an isotropic nearest neighbor interaction in the system. Further quantitative information is obtained by analyzing the dynamic susceptibility $\chi^{\prime \prime}(Q, E)$, given by

$$
S(Q, \omega)=\gamma_{0}^{2}\left(\frac{k_{i}}{k_{f}}\right) f(Q)^{2} \frac{1}{1-e^{-h \omega / k_{B} T}}\left(\frac{\chi^{\prime \prime}(Q, \omega)}{\pi}\right),
$$

where $\gamma_{0}^{2}=0.073 / \mu_{B}^{2}, k_{i}$ and $k_{f}$ represent initial and final neutron wave vectors, and $f(Q)$ is the form factor of magnetic ion (in this case Co ion). In Fig. 4(c), we plot $\chi^{\prime \prime}(Q, E)$ as a function of energy at $T=1.7 \mathrm{~K}$ at a representative $Q=$ $1.4 \AA^{-1}$. Clearly, a broad peak in $\chi^{\prime \prime}(Q, E)$, centered around $E \simeq 0.75 \mathrm{meV}$, is observed. At higher temperature, above $T_{c}$, the excitation becomes indistinguishable from the background, indicating the magnetic nature of inelastic peak. A dispersive excitation is detected at higher temperature, which can be associated to the phonon excitation in the system. However, the excitation does not prevail to high $q$ values, typical of phonon excitation. One explanation of such discrepancy may involve the coupling between phonon excitation and the dynamic magnetic interaction, which follows the magnetic form factor. Hence, the dispersive excitation disappears at higher $q$ value.

We have fitted the data using the random phase approximation (RPA) model [25]. Previously, the RPA model has been successful in describing inelastic phenomena in transition metal ion correlated systems [26,27]. Fitting using the RPA model is based on the assumptions that the only appreciable interaction is nearest neighbor interaction between Co ions, $J$, and the interaction is isotropic in nature. The small ordered moment, despite the long counting time, and a nearly $Q$ independent excitation in MACS measurement conform to the applicability of RPA model to estimate the exchange interaction between magnetic ions. Under RPA model, $\chi^{\prime \prime}(Q, E)$ is described by

$$
\chi^{\prime \prime}(Q, \omega)=\sum_{ \pm} \frac{\omega \chi_{0} \Gamma_{Q^{ \pm}}}{\Gamma_{Q^{ \pm}}^{2}+\omega^{2}}
$$

where $\Gamma_{Q^{ \pm}}=\Gamma\left[\begin{array}{lll}1 \mp \chi_{0} & J\end{array}\right]$ and $\chi_{0}$ is static susceptibility. Clearly, the RPA model describes the dynamic properties of CoSeAs very well. Fitted value of $J=0.25(4) \mathrm{meV}$ at $T=$ $1.7 \mathrm{~K}$ indicates a weak exchange interaction between Co ions. Obtained values of $\Gamma$ is plotted as a function of wave vector $Q$ in Fig. 4(d). $\Gamma$, representing the full width at half maximum of dynamic correlation or the inverse of relaxation time $\tau$, seems to be independent of the wave vector $Q$. The dynamic susceptibility at $T=135 \mathrm{~K}$ is barely distinguishable from the background. The small deviation from the background at low energy is most likely arising due to the paramagnetic fluctuation of Co ions at higher temperature.

The analysis of inelastic data reveals the large value of $\Gamma, \simeq 0.8 \mathrm{meV}$, for such a modest exchange coupled magnet. It suggests that Co ions are fluctuating with short relaxation time. Such behavior are usually observed in magnetically unstable systems [28]. Perhaps the stoichiometric composition of CoSeAs is on the verge of a transition to another phase of material.

\section{CONCLUSIONS}

In summary, we have synthesized another marcasite phase material CoSeAs. Neutron scattering investigation of polycrystalline CoSeAs reveals the development of long-range magnetic order below $T_{c}=36.2 \mathrm{~K}$. Given the fact that only a very few transition metal marcasites are known to manifest magnetic order, this is an important finding. Moreover, the weak nearest neighbor exchange coupling between Co ions makes it an interesting candidate material for the exploration of unconventional superconductivity using chemical doping method. Chemical pressure can further distort the anion octahedron, encompassing the transition metal ion, to induce a transition to another phase of material. Further research is highly desirable in this pursuit. Besides the exploration of a possible superconducting state in chemically doped CoSeAs, we envision two possible applications of this compound as photovoltaic absorber and in the design of robust electrocatalysts. There is an increasing trend to use marcasites and pyrites for photovoltaic application in recent years [6,29]. Future research on the study of optical properties of CoSeAs thin film can elucidate its possible application in photovoltaics. More recently, an analogous marcasite $\mathrm{CoSe}_{2}$ was demonstrated to preserve electrocatalytic integrity after long hours of usage in the acidic media [30]. Similar studies on the crystalline specimen of CoSeAs can reveal new electrocatalytic properties in this compound. 


\section{ACKNOWLEDGMENTS}

D.K.S. thankfully acknowledges the support by the Department of Energy, Office of Science, Office of Basic
Energy Sciences, under Grant No. DE-SC0014461. This work utilized facilities supported by the Department of Commerce.
[1] J. Zaanen, G. A. Sawatzky, and J. W. Allen, Phys. Rev. Lett. 55, 418 (1985).

[2] R. L. Carlin and A. J. van Duyneveldt, Magnetic Properties of Transition Metal Compounds (Springer, Berlin, 1977).

[3] D. Kitchaev and G. Ceder, Nat. Commun. 7, 13799 (2016)

[4] H. Holseth and A. Kjekshus, Acta Chem. Scand. 22, 3273 (1968).

[5] Y. Chen, S. Xu, Y. Li, R. J. Jacob, Y. Kuang, B. Liu, Y. Wang, G. Pastel, L. G. Salamanca-Riba, M. R. Zachariah, and L. Hu, Adv. Energy Mat. 71700482 (2017).

[6] K. Zhang, Z. Hu, X. Liu, Z. Tao, and J. Chen, Adv. Mater. 27, 3305 (2015).

[7] H. Li, X. Qian, C. Zhu, X. Jiang, Li Shaoa, and L. Hou, J. Mater. Chem. A 5, 4513 (2017).

[8] G. Hull and F. Hulliger, Nature (London) 220, 257 (1968).

[9] M. Amsler, S. Naghavi, and C. Wolverton, Chem. Sci. 8, 2226 (2017).

[10] J. B. Goodenough, A. Hamnett, G. Huber, F. Hullinger, M. Leib, S. K. Ramasesha, and H. Werheit, Physics of Nontetrahedrally Bonded Binary Compounds III (Springer, Verlag, 1984), Vol. 17.

[11] F. Hulliger, Nature (London) 198, 1081 (1963).

[12] B. Wilfong, X. Zhou, H. Vivanco, D. J. Campbell, K. Wang, D. Graf, J. Paglione, and E. Rodriguez, Phys. Rev. B 97, 104408 (2018).

[13] D. J. Campbell, L. Wang, C. Eckberg, D. Graf, H. Hodovanets, and J. Paglione, Phys. Rev. B 97, 174410 (2018).

[14] K. Selte and A. Kjekshus, Acta Chem. Scand. 25, 3277 (1971).

[15] NIST does not endorse any commercial product.

[16] P. Giannozzi, S. Baroni et al., J. Phys.: Cond. Matter 21, 395502 (2009).

[17] J. P. Perdew, K. Burke, and M. Ernzerhof, Phys. Rev. Lett. 77, 3865 (1996).

[18] M. Cococcioni and S. de Gironcoli, Phys. Rev. B 71, 035105 (2005).

[19] K. Momma and F. Izumi, J. Appl. Crystallogr. 44, 1272 (2011).
[20] L. Harriger, S. M. Disseler, J. Gunasekera, J. Rodriguez-Rivera, J. Pixley, P. Manfrinetti, S. K. Dhar, and D. K. Singh, Phys. Rev. B 95, 041102(R) (2017).

[21] D. K. Singh, A. Thamizhavel, S. Chang, J. W. Lynn, D. A. Joshi, S. K. Dhar, and S. Chi, Phys. Rev. B 84, 052401 (2011).

[22] G. Shirane, J. Tranquada, and S. Shapiro, Neutron Scattering with a Triple Axis Spectrometer: Basic Techniques (Cambridge University Press, New York, 2002).

[23] A. Dahal, Y. Chen, T. Heitmann, A. Thamizhavel, U. Paramanik, S. K. Dhar, and D. K. Singh, Phys. Rev. B 99 , 085135 (2019).

[24] See Supplemental Material at http://link.aps.org/supplemental/ 10.1103/PhysRevResearch.2.023168 for more information about the XRD refinement, Fermi surface nesting, magnetic form factor, and magnetic moment on individual atoms of CoSeAs.

[25] C. Broholm, J. K. Kjems, G. Aeppli, Z. Fisk, J. L. Smith, S. M. Shapiro, G. Shirane, and H. R. Ott, Phys. Rev. Lett. 58, 917 (1987).

[26] R. Birgeneau, H. Guggenheim, and G. Shirane, Phys. Rev. B 1, 2211 (1970).

[27] A. Dahal, J. Gunasekera, L. Harriger, S. H. Lee, Y. S. Hor, D. J. Singh, and D. K. Singh, Phys. Rev. B 94, 184516 (2016).

[28] B. G. Ueland, A. Kreyssig, K. Prokeš, J. W. Lynn, L. W. Harriger, D. K. Pratt, D. K. Singh, T. W. Heitmann, S. Sauerbrei, S. M. Saunders, E. D. Mun, S. L. Bud'ko, R. J. McQueeney, P. C. Canfield, and A. I. Goldman, Phys. Rev. B 89, 180403(R) (2014).

[29] L. Wu, N. Y. Dzade, L. Gao, D. O. Scanlon, Z. Öztürk, N. Hollingsworth, B. M. Weckhuysen, E. J. M. Hensen, N. H. de Leeuw, and J. P. Hofmann, Adv. Mat. 28, 9602 (2016).

[30] X.-L. Zhang, S.-J. Hu, Y.-R. Zheng, R. Wu, F.-Y. Gao, P.-P. Yang, Z.-Z. Niu, C. Gu, X. Yu, X.-S. Zheng, C. Ma, X. Zheng, J.-F. Zhu, M.-R. Gao, and S.-H. Yu, Nat. Commun. 10, 5338 (2019). 